OPEN ACCESS

Edited by:

Miguel López-Botet, Institut Hospital del Mar d'Investigacions Mèdiques,

Spain

Reviewed by: Karl-Johan Malmberg, Oslo University Hospital,

Norway

Francisco Borrego, BioCruces Health Research Institute, Spain

*Correspondence:

Ricciarda Galandrini ricciarda.galandrini@uniroma1.it;

Gabriella Palmieri gabriella.palmieri@uniroma1.it

these authors have contributed equally to this work.

¥These authors have contributed equally to this work as co-corresponding authors.

Specialty section: This article was submitted to NK and Innate Lymphoid Cell Biology, a section of the journal

Frontiers in Immunology

Received: 19 March 2018 Accepted: 24 April 2018 Published: 11 May 2018

Citation:

Capuano C, Battella S, Pighi C, Franchitti L, Turriziani O, Morrone $S$,

Santoni A, Galandrini R and Palmieri G (2018) Tumor-Targeting

Anti-CD20 Antibodies Mediate In Vitro Expansion of Memory Natural Killer Cells: Impact of CD16 Affinity Ligation Conditions and In Vivo Priming.

Front. Immunol. 9:1031 doi: 10.3389/fimmu.2018.01031

\section{Tumor-Targeting Anti-CD20} Antibodies Mediate In Vitro Expansion of Memory Natural Killer Cells: Impact of CD16 Affinity Ligation Conditions and In Vivo Priming

\author{
Cristina Capuano ${ }^{1 \dagger}$, Simone Battella ${ }^{1 \dagger}$, Chiara Pighi ${ }^{1}$, Lavinia Franchitti ${ }^{1}$, \\ Ombretta Turriziani ${ }^{2}$, Stefania Morrone ${ }^{1}$, Angela Santoni ${ }^{2,3,4}$, Ricciarda Galandrini ${ }^{1 * \neq}$ \\ and Gabriella Palmieri ${ }^{\text {** }}$

\begin{abstract}
${ }^{1}$ Department of Experimental Medicine, Sapienza University of Rome, Rome, Italy, ${ }^{2}$ Department of Molecular Medicine, Sapienza University of Rome, Rome, Italy, ${ }^{3}$ Laboratorio Pasteur Italia Fondazione Cenci Bolognetti, Sapienza University of Rome, Rome, Italy, ${ }^{4}$ IRCCS Neuromed, Pozzilli, Italy
\end{abstract}

Natural killer (NK) cells represent a pivotal player of innate anti-tumor immune responses. The impact of environmental factors in shaping the representativity of different NK cell subsets is increasingly appreciated. Human cytomegalovirus (HCMV) infection profoundly affects NK cell compartment, as documented by the presence of a CD94/ NKG2C+FceRl $\gamma$ - long-lived "memory" NK cell subset, endowed with enhanced CD16dependent functional capabilities, in a fraction of HCMV-seropositive subjects. However, the requirements for memory NK cell pool establishment/maintenance and activation have not been fully characterized yet. Here, we describe the capability of anti-CD20 tumor-targeting therapeutic monoclonal antibodies (mAbs) to drive the selective in vitro expansion of memory NK cells and we show the impact of donor' HCMV serostatus and CD16 affinity ligation conditions on this event. In vitro expanded memory NK cells maintain the phenotypic and functional signature of their freshly isolated counterpart; furthermore, our data demonstrate that CD16 affinity ligation conditions differently affect memory NK cell proliferation and functional activation, as rituximab-mediated low-affinity ligation represents a superior proliferative stimulus, while high-affinity aggregation mediated by glycoengineered obinutuzumab results in improved multifunctional responses. Our work also expands the molecular and functional characterization of memory NK cells, and investigates the possible impact of CD16 functional allelic variants on their in vivo and in vitro expansions. These results reveal new insights in Ab-driven memory NK cell responses in a therapeutic setting and may ultimately inspire new NK cell-based intervention strategies against cancer, in which the enhanced responsiveness to mAbbound target could significantly impact therapeutic efficacy.

Keywords: memory NK cells, cancer immunotherapy, therapeutic anti-CD20 mAb, CD16, in vitro expansion 


\section{INTRODUCTION}

The perspective of natural killer (NK) cells as exquisitely innate effectors is challenged by the recent appreciation that long-lasting NK cell populations with enhanced effector functions may arise in response to environmental factors, named adaptive or memory NK cells (1-3).

In vivo, memory NK cells have been identified in human cytomegalovirus (HCMV)-seropositive subjects, mainly on the basis of high expression levels of CD94/NKG2C activating receptor and CD57 terminal differentiation marker $(4,5)$. Recent studies have identified an immune-receptor tyrosine-based activation motif (ITAM)-bearing FceRI $\gamma$ adaptor protein-deficient $\left(\mathrm{Fc \varepsilon RI} \gamma^{-}\right)$NK cell subset in HCMV-seropositive individuals, endowed with a specific epigenetic signature and mostly overlapping with the CD94/NKG2C ${ }^{\text {high }}$ population (6-10). FceRI $\gamma$ chain deficiency thus represents the hallmark of memory NK cell population, together with variable loss of Syk and EAT2 signaling intermediates, low amount of the pro-myelocytic leukemia zinc finger (PLZF) transcription factor, and a distinctive genome-wide methylation profile similar to that of memory effector $\mathrm{T}$ cells $(8,9)$. As a consequence of FceRI $\gamma$ deficiency, memory NK cells exhibit lower surface levels of FceRI $\gamma$-dependent NKp46 and NKp30 activating receptors, with respect to FceRI $\gamma^{+}$conventional NK cells $(1,6)$.

Memory NK cells can constitute up to $70 \%$ of the total NK cell population in HCMV-seropositive individuals. The mechanistic basis of their selective expansion is incompletely understood. It has been proposed that primary HCMV infection drives the priming and proliferation of memory NK cells in a NKG2Cdependent manner $(1-3,11,12)$. Such expansion may be maintained by a variety of different viral super-infections. In particular, an expanded population of memory NK cells was detected in EBV-, HBV-, HCV-, and HIV-seropositive individuals, only when patients were also seropositive for $\operatorname{HCMV}(13,14)$. It is therefore conceivable that $\mathrm{Ab}$-mediated immune responses may drive the proliferation and maintenance of an already existing pool of memory NK cells, in some viral disease settings. Seminal in vitro studies offered a mechanistic explanation for the role of virus specific Abs in sustaining memory NK cell expansion, establishing a pivotal role for $\mathrm{CD} 16$ binding to $\mathrm{Ab}$-opsonized infected cells $(8,9)$.

CD16, the low-affinity $\mathrm{Fc}$ receptor for IgG, or FcyRIIIa, represents a prototype NK activating receptor; its engagement by IgG-opsonized targets is sufficient to trigger antibody-dependent cytotoxicity (ADCC), as well as the production of pro-inflammatory cytokines and chemokines, such as IFN- $\gamma$, TNF $\alpha$, IL-6, GM-CSF, and CCL5 $(15,16)$. In particular, NK-derived IFN- $\gamma$ stands as a well-recognized key immunoregulatory factor in the shaping of anti-tumor adaptive immune responses, by modulating dendritic cells (DCs) and T-cell responses $(17,18)$. Moreover, the capability of CD16-initiated signals to regulate NK cell proliferation and death, under selective conditions, has been also shown $(19,20)$.

Human CD16 exhibits two extracellular Ig domains, a short cytoplasmic tail and a transmembrane domain that enables its association with ITAM-containing $\mathrm{CD} 3 \zeta$ and FceRI $\gamma$ chains (21), which guarantee Syk- and ZAP-70-dependent signal transduction (16).

Multiple lines of evidence highlighted a functional superiority of memory compared with conventional NK cells, in response to stimulation through CD16, particularly in terms of cytokine production $(6-8,22)$. Indeed, memory NK cells exhibit a greatly enhanced ability to produce IFN- $\gamma$, as a consequence of hypomethylated IFNG regulatory region (23), in response to activation via $\mathrm{CD} 16$, thus providing a prompt and powerful response against antibody-opsonized target cells.

The exploitation of memory NK cells in cancer combination immunotherapy may be highly attractive, because of their unique properties of CD16-dependent longevity and amplified functional responses. Indeed, CD16-triggered ADCC and phagocytosis, performed by NK cells and macrophages, respectively, are among the main immune-dependent mechanisms by which therapeutic monoclonal antibodies (mAbs) mediate tumor cell killing (24-27). Moreover, CD16-dependent immunomodulatory activity may contribute to the "vaccinal effect" of therapeutic tumor-targeting $\mathrm{mAbs}$, i.e., the promotion of adaptive anti-tumor immune responses that confer long-term protection $(17,18$, $28,29)$. This concept is supported by the evidence that a single nucleotide polymorphism of the FCGR3A gene (c.559G >T, p.Phe158Val), encoding for a lower (Fc $\gamma$ RIIIA-158F) or a higher (FcrRIIIA-158V) affinity allele of CD16 receptor, affects the clini$\mathrm{cal}$ response to rituximab anti-CD20 $\mathrm{mAb}$ treatment that stands as a well-established first-line therapeutic option in several B cell malignancies $(30,31)$. More recently, new mAbs with enhanced affinity for CD16 have been generated. Among them, obinutuzumab, recently approved for clinical use (32-34), is a type II glycoengineered anti-CD20 mAb with an afucosylated crystallizable fragment $(\mathrm{Fc})$ domain that binds to a $\mathrm{CD} 20$ epitope in a different space orientation and with a wider elbow-hinge angle with respect to the reference molecule rituximab (35).

Our recent data highlighted that distinct CD16 aggregation conditions, obtained through sustained contact with target cells opsonized by different anti-CD20 mAbs, differently promote the shift of NK cell functional program $(36,37)$.

Here, we address the capability of anti-CD20 mAbs to affect memory NK cell dynamics. We demonstrate that the co-culture with anti-CD20 mAb-opsonized targets selectively supports the in vitro expansion of in vivo primed memory NK cells, which phenotypically and functionally mirror their freshly isolated counterpart. CD16 engagement under quantitatively different affinity ligation conditions qualitatively impacts on memory NK-cell responses, being rituximab more efficient in supporting expansion, and obinutuzumab more active in inducing functional activation.

We also investigate the possible impact of CD16 functional allelic variants on memory NK cell in vivo and in vitro expansion.

\section{MATERIALS AND METHODS}

\section{Cell Systems and Anti-CD20 mAbs}

Peripheral blood mononuclear cells (PBMCs) were freshly isolated from peripheral blood samples of anonymized healthy donors of 
Transfusion Center of Sapienza University of Rome, over a FicollHypaque (Cedarlane) density gradient. Written informed consent was obtained from blood donors, and both the informed consent form and procedure were approved by the Ethics Committee of Sapienza University of Rome. The study was conducted in accordance with the Declaration of Helsinki. The following human cell lines were used as targets: Raji CD20 $0^{+}$lymphoblastoid, provided by Dr. F. D. Batista (Cancer Research UK, London), and K562 erithroleukemia, obtained from ATCC. All cell lines were kept in culture for less than 2 consecutive months in $10 \%$ fetal calf serum (FCS)- and 1\% L-glutamine (both from Euroclone)-containing RPMI 1640 and regularly checked for mycoplasma presence. The following anti-CD20 mAbs were used: the chimeric IgG1к type I

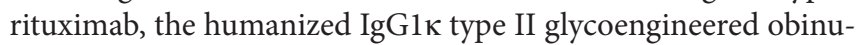
tuzumab (GA101), and its nonglycoengineered parental molecule (GA101-WT), all kindly provided by Dr. Christian Klein, Roche Innovation Center Zurich (Schlieren, Switzerland).

\section{Immunostaining and Cytofluorometric Analysis \\ Phenotypic Characterization of NK Cells}

Peripheral blood-derived and in vitro cultured mononuclear cell populations were identified by a combination of physical parameters and immunostaining with saturating concentrations of the following fluorochrome-conjugated mAbs: anti-CD3 PerCP (clone: SK7, cat \#: 347344; BD Biosciences) or PerCP-Vio700 (clone: REA613, cat \#: 130-109-465; Miltenyi Biotec), anti-CD56 APC (clone: B159, cat \#: 555518; BD Biosciences) or APC-Vio770 (clone: REA 196, cat \#: 130-100-694; Miltenyi Biotec), CD16 PE (clone: B73.1, cat \#: 347617; BD Biosciences) or PE-Vio770 (clone: REA423, cat \#: 130106-706; Miltenyi Biotec), anti-FceRI $\gamma$ subunit FITC (polyclonal antibody, cat \#: FCABS400F; Merck), anti-NKp46 APC (clone: REA808, cat \#: 130-112-122; Miltenyi Biotec), anti-NKG2C PE (clone: 134591, cat \#: FAB138P; R\&D Systems), anti-PLZF PE (clone: Mags.21F7, cat \#: 12-9320-82; ThermoFisher Scientific), anti-PD-1 PE (clone: EH12.2H7, cat \#: 329906; BioLegend), and anti-PD-L1 APC (clone: 29E.2A3, cat \#: 329708; BioLegend). Samples were stained for surface antigens for $30 \mathrm{~min}$ at $4^{\circ} \mathrm{C}$, washed with PBS (Euroclone) containing 2\% FCS and 2 mM EDTA (used for all washing steps), fixed with $2 \%$ paraformaldehyde for $20 \mathrm{~min}$ at room temperature (RT), washed, permeabilized with washing solution supplemented with $0.05 \%$ Triton-X 100 for $30 \mathrm{~min}$ at RT, and stained for intracellular antigens for $30 \mathrm{~min}$ at $4^{\circ} \mathrm{C}$.

\section{Characterization of Fc $\gamma$ RIIIA-158V/F Allotype}

The FcyRIIIA-158V/F allotype was determined on a cohort of 217 donors, as previously described (37). Briefly, PBMCs were stained with APC-conjugated anti-CD56 $\mathrm{mAb}$ and the following anti-CD16 mAbs: FITC-conjugated 3G8 (cat \#: 555406; $\mathrm{BD}$ Biosciences) that binds to a not polymorphic epitope or FITC-conjugated MEM-154 (cat \#: MAB-1457F; Immunological Science), whose binding to CD16 is dependent on the presence of valine (38). The ratio between the mean fluorescence intensity (MFI) of MEM-154 and 3G8 allows to classify the following three different phenotypes: F/F (ratio < 0.04), V/V (ratio >0.62), and $\mathrm{V} / \mathrm{F}$ (ratio between 0.15 and 0.48 ).

\section{CD107a Mobilization and Intracellular IFN- $\gamma$ Production}

Freshly isolated PBMCs and their in vitro cultured counterparts were stimulated with Raji cells (2:1), opsonized or not with the minimum saturating dose of rituximab $\left(1 \mu \mathrm{g} / 1 \times 10^{6}\right)$ or obinutuzumab $\left(0.1 \mu \mathrm{g} / 1 \times 10^{6}\right)$, or with $\mathrm{K} 562$ targets, for $6 \mathrm{~h}$ at $37^{\circ} \mathrm{C}$ in the presence of PE-conjugated anti-CD107a mAb (clone: H4A3, cat \#: 555801; BD Biosciences) and $50 \mu \mathrm{M}$ Monensin (Golgi-stop; cat \#: M5273; Merck). After the first hour, $10 \mu \mathrm{g} / \mathrm{ml}$ Brefeldin A (cat \#: B7651; Merck) was added. At the end of stimulation, cells were washed with $5 \mathrm{mM}$ EDTA containing PBS and then stained as earlier, adding the anti-IFN- $\gamma$ APC (clone: B27, cat \#: 554702; BD Biosciences) after permeabilization.

Samples were analyzed with a FACSCanto II (BD), and data were obtained with FlowJo vX.0.7 (TreeStar) software. Where required, twin samples were added and stained with isotype control $\mathrm{mAb}$, used to set the threshold for antigen positivity. The values of cell counts and cytofluorimetric analysis percentages were used to obtain the absolute number of different NK cell subsets.

\section{In Vitro NK Cell Culture}

Freshly isolated PBMC from healthy donors were seeded in round-bottomed 96-well plates (50,000 cells/well) and cultured for 10 days in RPMI 1640 medium supplemented with $10 \%$ FCS, $1 \%$ L-glutamine, $1 \%$ penicillin/streptomycin, and $100 \mathrm{U} /$ $\mathrm{ml}$ of human recombinant IL-2 (cat \#: AF-200-02; Peprotech); after 2 days, irradiated Raji cells (3000 rad), incubated or not with an excess of rituximab, obinutuzumab, or wt-obinutuzumab (GA101-WT) (all from Roche) for $20 \mathrm{~min}$ at RT, were washed and added to the cultures (25,000 cells/well). Recombinant hIL-2-containing medium (100 IU/ml) was half-replaced every 2 days. At day 10, cells were harvested and used for phenotypic and functional analyses. For functional assays, $10 \mathrm{ng} / \mathrm{ml}$ of IL-15 (cat \#: AF-200-15; Peprotech) was added at day 7.

\section{HCMV Serostatus Analysis}

Anti-HCMV plasma IgG titers were determined by CMV IgG Immulite 2000 System (Siemens Healthineers), according to the manufacturer's instructions.

\section{Statistical Analysis}

Differences between two groups were determined by two-tailed Mann-Whitney $U$ test or Wilcoxon signed rank test, as appropriate. Analyses were performed using Prism v.6 (GraphPad Software) and SPSS v24.0 (IBM Italia SpA) software packages. Differences were considered to be statistically significant when $p$ value was $<0.05$ (two sided).

\section{RESULTS}

\section{In Vivo Characterization of Memory NK Cell Compartment: Impact of FCGR3A- V158F Polymorphism}

Our analysis of an extended cohort of 275 healthy donors evidenced that a discrete memory NK cell population, identified as $\mathrm{CD}^{-} \mathrm{CD} 56^{\mathrm{dim}} \mathrm{CD} 16^{+} \mathrm{FceRI} \gamma^{-}$(Figure 1A), and accounting 


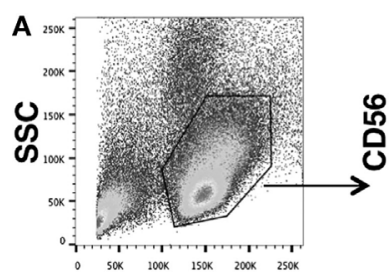

FSC

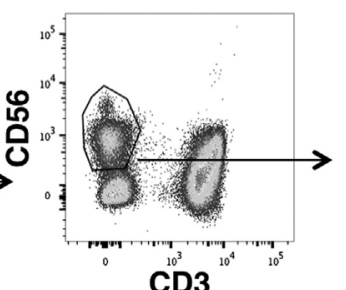

CD3

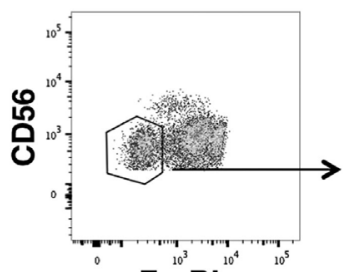

FCeRly

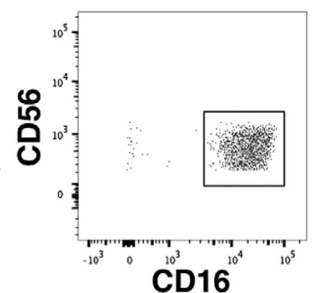

CD16

CD3-CD56 ${ }^{\text {dim }}{ }^{-}$16 $+F c \varepsilon R l \gamma-$

B

CD3-CD56+

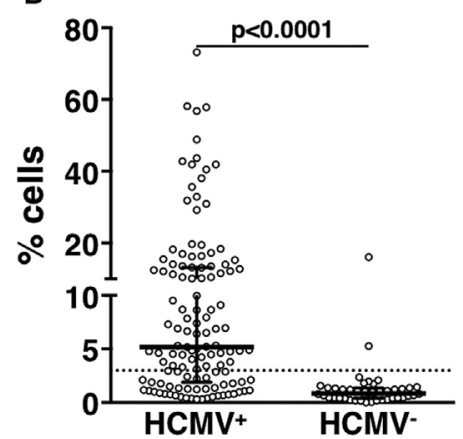

D

HCMV+ HCMV

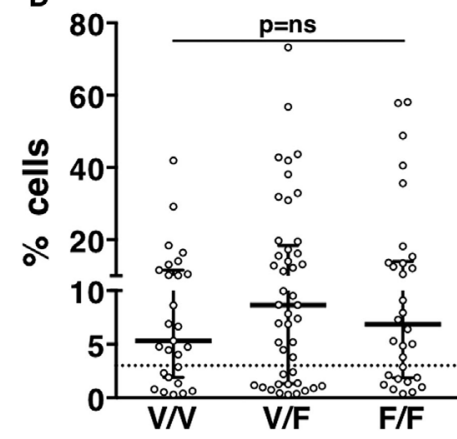

E
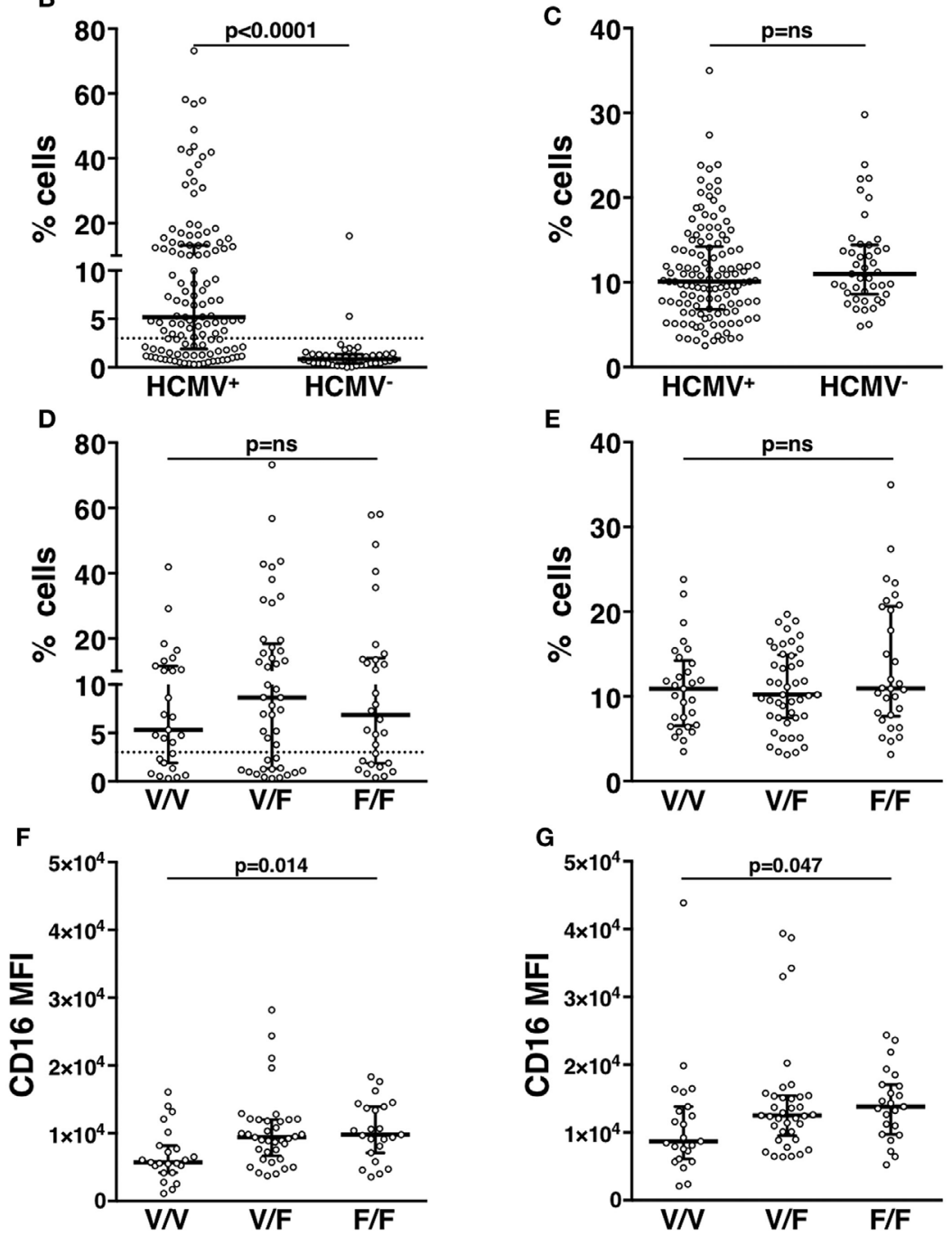

G

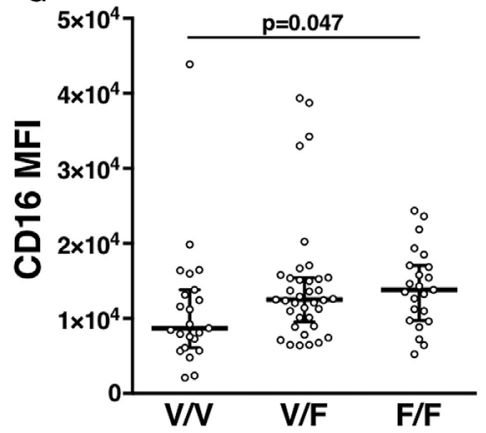

FIGURE 1 In vivo characterization of human memory natural killer (NK) cells. (A) Gating strategy for the identification of CD3-CD56 dim $C D 16^{+}$FceRl $\gamma^{-}$memory NK cells. (B) Percentage of memory NK cells (with respect to total NK cells) in the peripheral blood of human cytomegalovirus (HCMV) seropositive (HCMV $\left.{ }^{+}\right)$and seronegative $\left(\mathrm{HCMV}^{-}\right)$healthy donors; dotted line marks the value of $3 \%$. (C) Percentage of total $\mathrm{CD}^{-}{ }^{-} \mathrm{CD}^{2} 6^{+} \mathrm{NK}$ cells among peripheral blood mononuclear leukocytes, in the same individuals. (D) Percentage of memory NK cells (with respect to total NK cells) in the peripheral blood of HCMV+ healthy donors, stratified according to CD16 allotype; dotted line marks the value of 3\%. (E) Percentage of total CD3-CD56 ${ }^{+}$NK cells among peripheral blood mononuclear leukocytes, in the same individuals. CD16 mean fluorescence intensity (MFI) on memory (F) and total (G) NK cells of HCMV+ healthy donors, stratified according to CD16 allotype. Bars represent median and 10-90 percentiles. $p$ values of pairwise comparisons are from Mann-Whitney non-parametric test.

for more than $3 \%$ of total $\mathrm{CD}^{-} \mathrm{CD} 56^{+} \mathrm{NK}$ cells, was present in $52.4 \%$ of individuals (data not shown). In line with the literature (8-10), the presence of memory NK cells strictly correlated with HCMV seropositivity, as shown in serotyped individuals (Figure 1B). On the contrary, the percentage of total NK cells in $\mathrm{HCMV}$-seropositive and -seronegative individuals was not 
significantly different (Figure 1C). FceRI $\gamma^{-}$cells expressed other markers previously associated to memory NK cell profile $(6,8,9)$ : the percentage of $\mathrm{NKG}_{2} \mathrm{C}^{+}$cells was higher whereas the fraction of $\mathrm{NKp}_{4} 6^{+}$cells was lower, with respect to the FceRI $\gamma^{+}$counterpart. Moreover, PLZF transcription factor and CD16 expression levels were significantly lower in memory with respect to conventional NK cells (Figures S1A-D in Supplementary Material). Additionally, memory NK cells expressed PD-L1 at significantly lower levels than their conventional counterpart (Figure S1E in Supplementary Material).

It has been proposed that in vivo maintenance of a longlasting memory NK cell population relies on CD16-dependent interaction with antiviral Ab-opsonized infected cells (7, 9). In order to analyze the impact of CD16 affinity for IgG on the in vivo development of memory $\mathrm{NK}$ cell compartment, $\mathrm{HCMV}^{+}$ individuals were stratified on the basis of CD16 allotype: V/V (homozygous for high-affinity allele, 29 donors), F/F (homozygous for low-affinity allele, 29 donors), and V/F (45 donors). The percentage of total NK cells and that of memory NK population were not significantly different among individuals bearing different CD16 allotypes (Figures 1D,E), although V/V donors displayed a tendentially reduced frequency of memory NK cells (Figure 1D). Notably, both memory (Figure 1F) and total (Figure 1G) NK cells of V/V donors expressed lower levels of CD16 receptor, with respect to F/F donors. Nevertheless, the reduced CD16 levels of memory NK cells with respect to conventional NK cells (Figure S1A in Supplementary Material) were maintained across the three CD16 allotypes (Figure S2 in Supplementary Material).

\section{Anti-CD20-Mediated CD16 Ligation Induces the Selective In Vitro Expansion of Memory NK Cells: Impact of HCMV Seropositivity Status and CD16 Affinity Ligation Conditions}

Previous work has demonstrated that in vitro expansion of memory NK cells critically requires Ab-opsonized virus-infected cells $(7,9)$. In order to assess whether therapeutic tumor-targeting mAbs may drive memory NK cells expansion, we co-cultured healthy donor PBMC that displayed a sizeable population of memory ( $>3 \%$ of NK cells), with Raji lymphoblastoid B cell line, opsonized or not with rituximab anti-CD20 mAb, in the presence of IL-2 (Figure 2A). While conventional NK cells efficiently underwent expansion (up to 50-fold, Figure 2C), independently from rituximab, memory NK cells proliferated much more markedly in the presence of rituximab (4.6-76.3-fold) than in its absence (0.1-16.7-fold) (Figure 2B), indicating an antibody requirement to support memory NK cell expansion.

In order to assess whether these in vitro culture conditions could promote memory NK cell generation, or rather amplify a pre-existing memory NK cell pool driven by in vivo HCMV exposure, we assayed memory NK cell expansion dynamics in donors that lacked a sizeable memory population (less than $3 \%$ of total NK cell population), obtained from either HCMV-seropositive and -seronegative individuals. Memory NK cells more vigorously proliferated in the presence of rituximab than in its absence, when derived from $\mathrm{HCMV}^{+}$donors. At variance, rituximab addition did not appreciably affect in vitro expansion of memory NK cells from HCMV-seronegative individuals (Figure 2D). No significant differences in the extent of conventional NK cell subset proliferation were observed between $\mathrm{HCMV}^{+}$and $\mathrm{HCMV}^{-}$ samples, either in the presence or in the absence of rituximab (Figure 2E).

Collectively, these data indicate that memory NK cells selectively expand in vitro in a rituximab-dependent manner and that such response critically requires a previous HCMV in vivo priming.

We then compared three anti-CD20 mAbs, endowed with low [rituximab or obinutuzumab wild-type, fucosylated parental $\mathrm{mAb}$ (GA101-WT)] or high (obinutuzumab) binding affinity for CD16, for their ability to drive memory NK cell proliferation; in this system, we could dissect the impact of FcR binding affinity from that of targeted epitope and binding orientation on CD20 molecule. Rituximab was markedly more efficient in inducing the expansion of memory NK cells, as compared with obinutuzumab; Fc glycoengineering only partially accounted for such difference, since the expansion capability of GA101-WT was intermediate, suggesting that factors other than CD16 affinity may impact on memory NK cell expansion (Figure 3A). Moreover, as conventional NK cell proliferation was negatively affected by obinutuzumab, it is possible that signals arising under high CD16 affinity ligation conditions may interfere with NK cell expansion (Figure 3B). However, T-cell proliferation was not negatively affected by obinutuzumab presence, in the same samples (data not shown).

Memory NK cells expressing all CD16 allotypes expanded in response to rituximab, with higher levels in low-affinity $\mathrm{F} / \mathrm{F}$, with respect to V/F individuals. Furthermore, obinutuzumab resulted less efficient in inducing memory NK cell proliferation independently from CD16 allotype, confirming its ability to overcome the affinity ligation differences due to CD16 polymorphism (Figure 3C).

\section{Phenotypic and Functional Profile of In Vitro Expanded Memory NK Cells}

Consistent with what observed in the freshly isolated population (Figure S1 in Supplementary Material), in vitro cultured memory NK cells displayed a lower percentage of NKp46 cells, as compared to $\mathrm{Fc \varepsilon RI} \gamma^{+}$conventional population; the frequency of $\mathrm{NKG}_{2} \mathrm{C}^{+}$memory NK cells remained significantly higher with respect to conventional ones; and similar to what observed in freshly isolated samples, the expression levels of PLZF transcription factor and CD16 were lower in cultured memory NK cells, as compared to conventional NK cells (Figures 4A-D). However, it must be noted that the expression of all these markers underwent marked modulation upon in vitro culture, on both memory and conventional NK cell subsets (compared with the respective panels of Figure S1 in Supplementary Material). Interestingly, PD-L1 expression stably remained on a low percentage of memory NK cells, while significantly declined on conventional NK cells, upon in vitro expansion (Figure 4E; Figure 1E in Supplementary Material). 

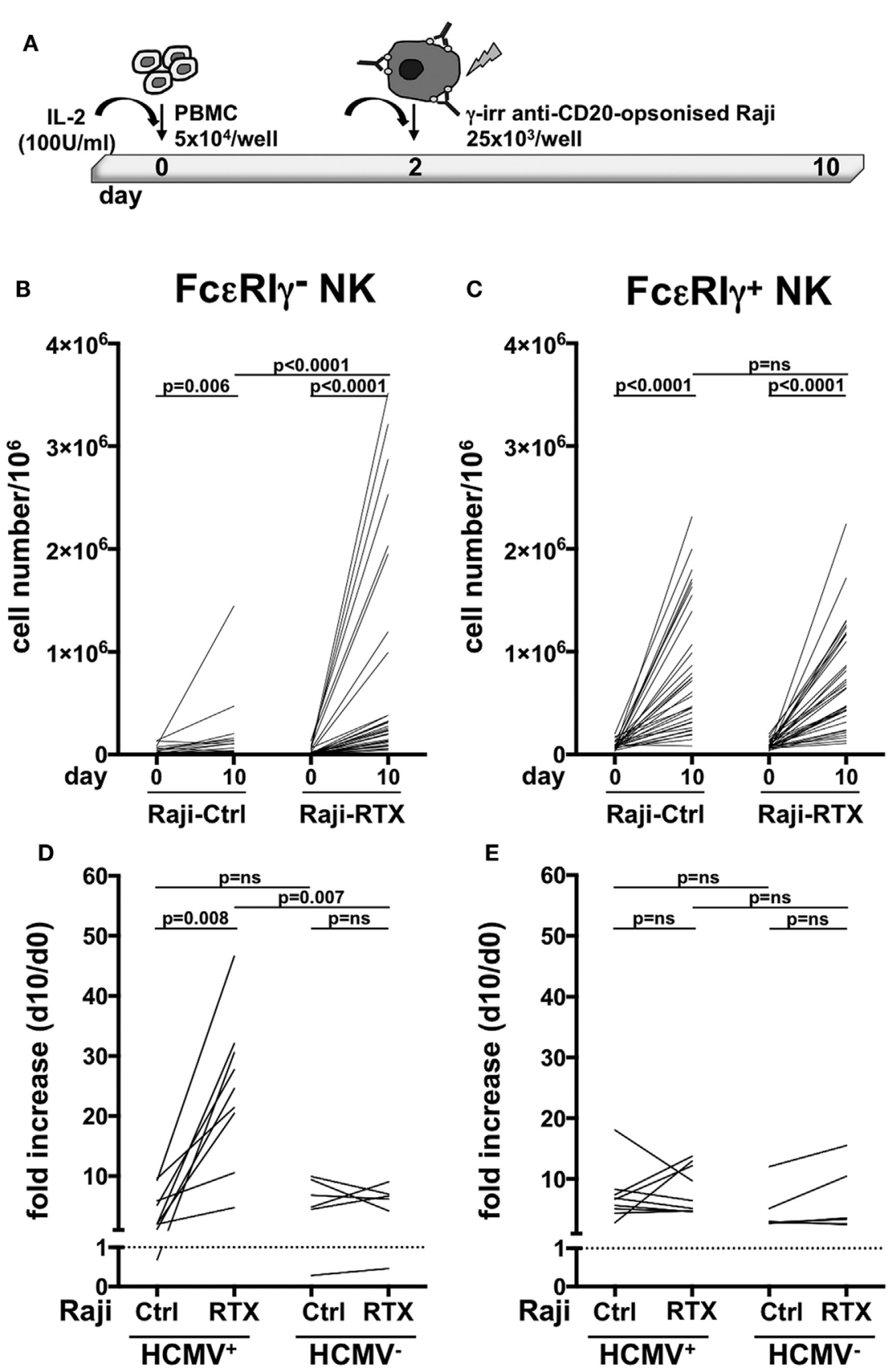

FIGURE 2 | In vitro memory natural killer (NK) cell expansion upon interaction with rituximab-opsonized Raji lymphoblastoid cells. (A) Experimental setting of NK cell co-culture protocol. (B) Memory (FceRl $\gamma^{-}$) and (C) conventional (FceRl $\left.\gamma^{+}\right)$CD3 $^{-}$CD56 dim CD16 ${ }^{+}$NK cell numbers (per million mononuclear cells), at the start (day 0) and at the end (day 10) of co-culture with Raji cells, opsonized (RTX) or not (Ctrl) with rituximab anti-CD20 monoclonal antibody (mAb).

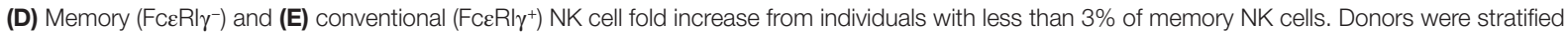
according to human cytomegalovirus (HCMV) seropositivity status. Dotted line marks the value of 1. $p$ values of pairwise comparisons are from Wilcoxon and Mann-Whitney non-parametric tests, as appropriate.

The ability to mediate enhanced functional responses to $\mathrm{Ab}$ stimulation, particularly in terms of IFN- $\gamma$ production, is a wellestablished characteristic of memory NK cells $(6,7,9,22)$.

We assessed memory NK cell responsiveness to CD16 or to direct target stimulation. In freshly isolated populations, upon CD16 stimulation obtained with rituximab or obinutuzumab-opsonized targets, the frequency of memory NK cells able to mediate both degranulation and IFN- $\gamma$ production was significantly higher with respect to the conventional counterpart. Notably, obinutuzumabmediated stimulation resulted more efficient in the induction of multifunctional responses in both memory and conventional NK cells, with respect to rituximab stimulation (Figure 5A). 

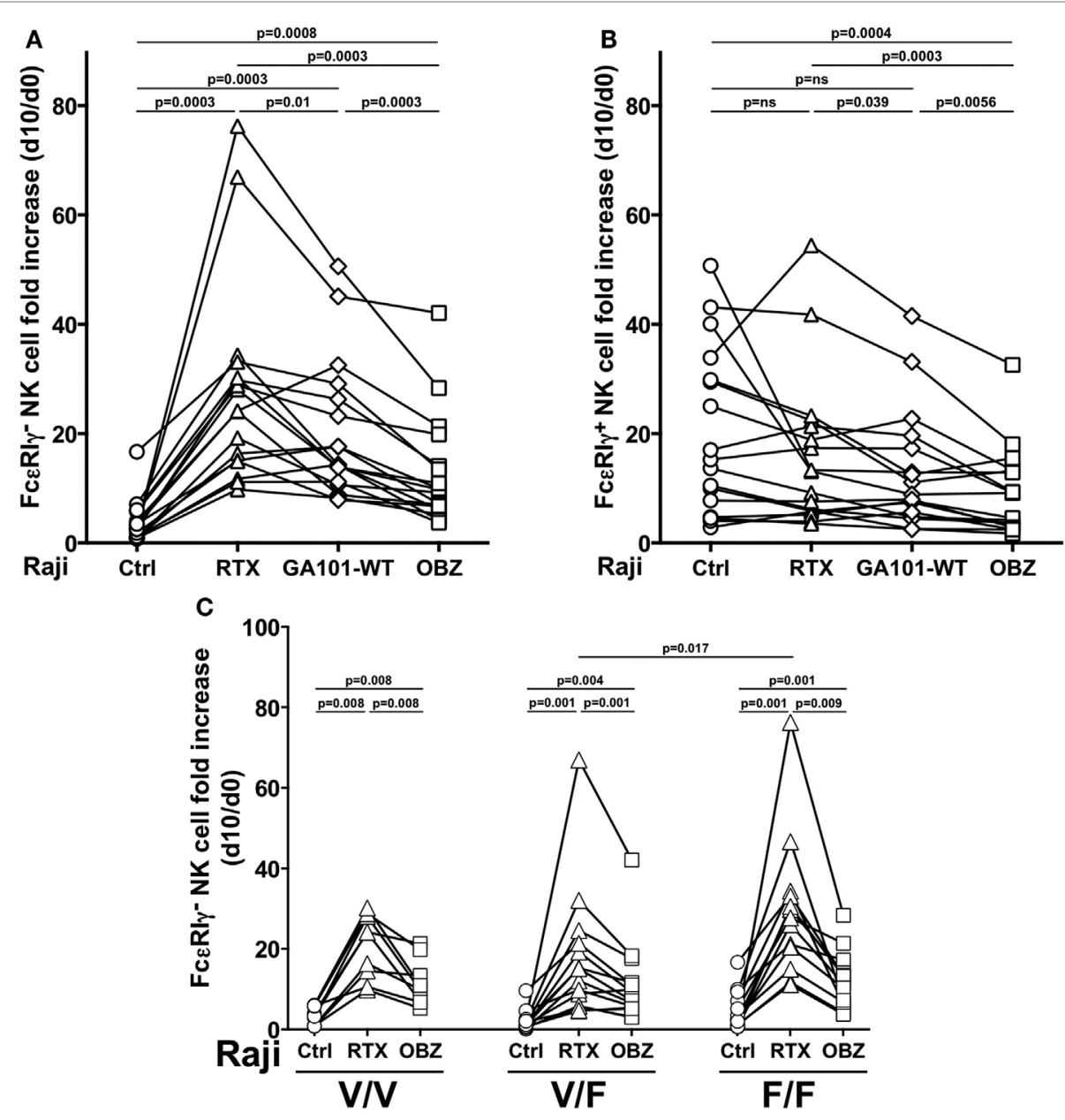

FIGURE 3 Effect of CD16 affinity ligation conditions on memory natural killer (NK) cell in vitro expansion from $\mathrm{HCMV}^{+}$individuals. (A) Memory (FcERl $\left.\gamma^{-}\right)$and (B) conventional ( $\left(\mathrm{Cc} \varepsilon \mathrm{R} / \gamma^{+}\right) \mathrm{CD} 3^{-} \mathrm{CD} 56^{\mathrm{dim}} \mathrm{CD} 16^{+} \mathrm{NK}$ cell fold increase, in response to the presence of Raji opsonized, or not (Ctrl), with rituximab (RTX), glycoengineered obinutuzumab (OBZ), or wild-type OBZ (GA101-WT). (C) Memory NK cell fold increase in response to rituximab or glycoengineered OBZ in samples stratified according to donor's CD16 allotype; Only statistically significant comparisons are depicted. Donors reported in Figures $\mathbf{2 B}-\mathbf{C}$ are included. $p$ values of pairwise comparisons are from Wilcoxon and from Mann-Whitney non-parametric tests, as appropriate.

Basal responsiveness of in vitro expanded NK cells was enhanced, as a consequence of the exposure to IL-2 plus IL-15 during the last 3 days of culture. In these conditions, obinutuzumab-mediated stimulation was able to induce a significantly higher response rate in memory with respect to conventional NK cells (Figure 5B). Differently, memory NK cell responses to rituximab-coated target cells were only tendentially higher than conventional NK cells, possibly in dependence on the markedly low CD16 expression level (see Figure 4A). Furthermore, in line with the literature and similarly to what observed on their fresh counterparts, mAb-expanded cultured memory NK cells displayed a defective ability to respond to K562 target cells (6).

\section{DISCUSSION}

Natural killer cell's role in tumor immunosurveillance, as well as their involvement in immune-dependent therapeutic efficacy of tumor-targeting mAbs, is widely recognized $(27,39)$. NK cell functional and phenotypic heterogeneity, together with the recent identification of a long-lived and hyper-responsive subset, named adaptive or memory, have raised much interest, to the end of a better exploitation of this anti-tumor effector in the development of innovative combination immunotherapeutic strategies.

The present work provides a further characterization of freshly isolated human memory NK cells, identified as $\mathrm{CD}^{-}{ }^{-} \mathrm{CD} 56^{\mathrm{dim}} \mathrm{CD} 16^{+} \mathrm{FceRI} \gamma^{-}$, and defines the ability of therapeutic mAb-coated tumor cells to efficiently drive their in vitro expansion from HCMV-seropositive healthy donors' PBMC. Indeed, we developed an effective in vitro culture system, consisting of a 10-day co-culture of PBMC with irradiated lymphoblastoid Raji cells opsonized with anti-CD20 mAbs, in IL-2-containing medium. Our results demonstrate the strict dependence of memory NK cell proliferation on the presence of opsonizing $\mathrm{mAb}$, as their expansion in the presence of not opsonized targets was only marginal. These data strongly support the concept that CD16-initiated signals are crucially involved in triggering 

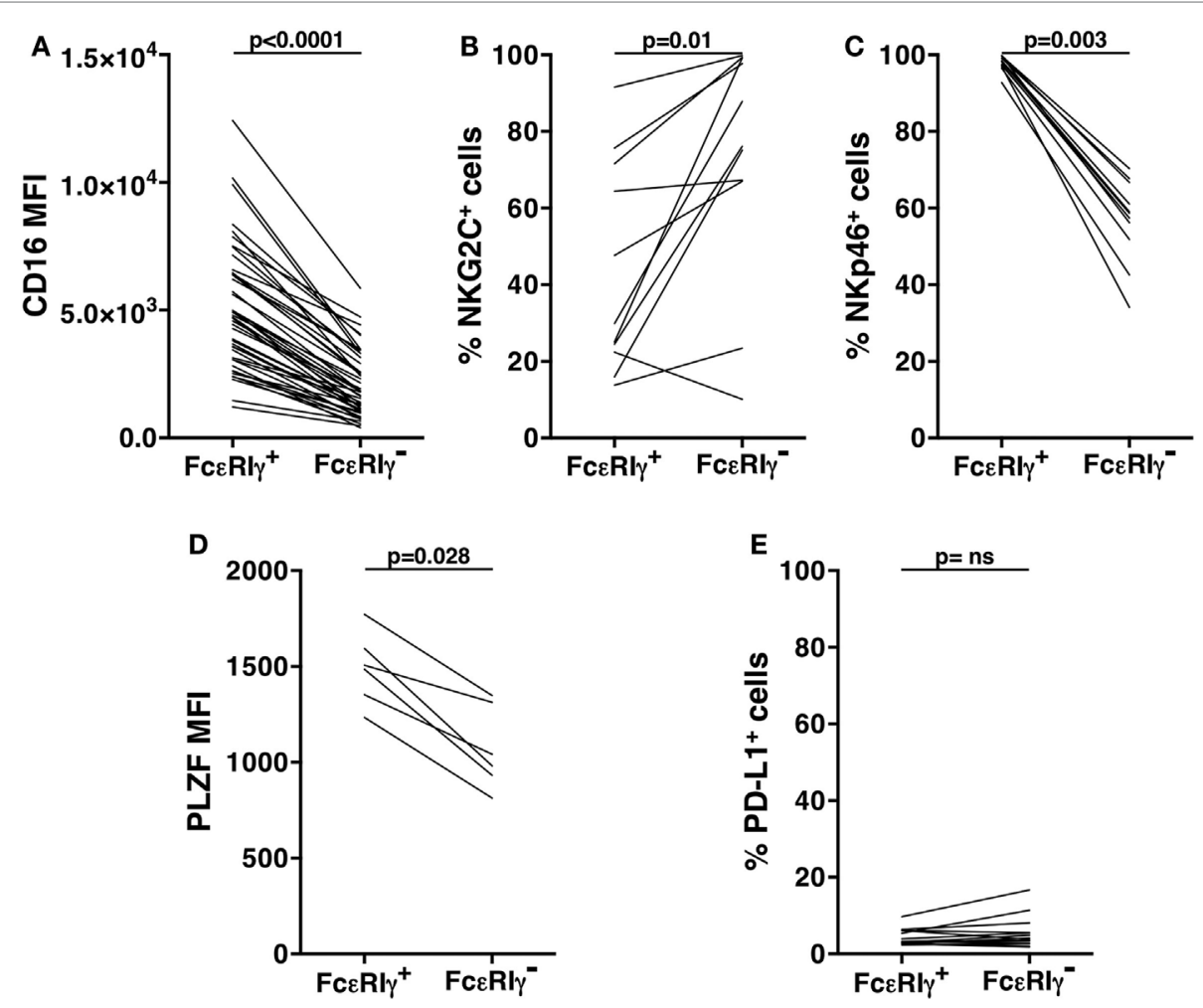

FIGURE 4 | Phenotypic characterization of in vitro cultured human memory natural killer (NK) cells. CD16 mean fluorescence intensity (MFI) (A), frequency of $\mathrm{NKG}_{2} \mathrm{C}^{+} \mathbf{( B )}$, and of NKp46+ (C) cells, pro-myelocytic leukemia zinc finger (PLZF) MFI (D), and percentage of PD-L1+ (E) cells, were evaluated on memory (FCeRly ${ }^{-}$) and conventional (FceRl $\gamma^{+}$) CD3-CD56 ${ }^{\text {dim }} \mathrm{CD}^{-} 6^{+} \mathrm{NK}$ cells, upon co-culture with rituximab-opsonized Raji cells. $p$ values of pairwise comparisons are from Wilcoxon non-parametric test.

the proliferation of this specific subset. In accordance, previous evidence had shown the capacity of anti-viral IgG-opsonized cells to induce memory NK cells proliferation $(8,9)$. Differently, conventional NK cells were capable of effectively and comparably expand upon co-culture with either not opsonized or therapeutic mAb-coated feeder cells, thus suggesting that cell-cell interactions with surface ligands expressed by Raji lymphoma cells, together with cytokines, provide sufficient proliferative signals to them. Indeed, the capability of $\mathrm{EBV}^{+}$lymphoblastoid cell lines to induce NK cell proliferation has been widely reported (40), and the crucial role of 2B4/CD48 interaction in this context has been recently proposed (41). Moreover, CD16-dependent NK cell proliferation has been recently reported, in a 21 -day culture protocol under selected co-stimulation conditions, but no evaluation of the memory NK cell compartment was performed (20). It is conceivable that CD16-dependent memory NK cell proliferation also relies on multiple accessory signals, conveyed by cell-cell contacts and soluble mediators; in this context, the synergistic effect of antisera and virus-infected cells was reported $(8,9)$. In our system, ligands expressed by Raji lymphoblastoid B cells may provide accessory proliferative signals to memory NK cells; among them, CD2 ligand, CD58, has been shown to co-stimulate memory NK cell responses (22). Moreover, monocyte-derived IL-12, probably stimulated through $\mathrm{Fc} \gamma \mathrm{R}$ engagement by antiCD20-opsonized targets, likely mediates a critical contribution through the up-regulation of CD25, as demonstrated by a recent report (12).

Our results identify donor $\mathrm{HCMV}$-seropositive status as a prerequisite to allow anti-CD20 mAb-dependent memory NK cell in vitro expansion. In fact, in vitro memory $\mathrm{NK}$ cell proliferation could be obtained from $\mathrm{HCMV}^{+}$, but not from $\mathrm{HMCV}^{-}$donors that in vivo lacked a sizeable ( $>3 \%$ of total NK cells) memory NK cell population. These data suggest that HCMV exposure is indispensable for the priming of a memory NK cell pool and are in accordance with multiple lines of evidence that have demonstrated the unique ability of HCMV to epigenetically shape a $\mathrm{NKG}_{2} \mathrm{C}^{+}$memory NK compartment, and the capability of viral reactivation to promote a long-lasting expansion of the memory NK cell pool (1-5). Furthermore, in vitro expansion of $\mathrm{NKG}^{+} \mathrm{C}^{+}$ memory NK cells could be achieved by co-culturing NK cells with HCMV-infected fibroblasts or HLA-E-expressing feeder $(11,12,42)$. In these conditions, the interaction between the activating receptor CD94/NKG2C and its cellular ligand HLA-E, in combination with inflammatory cytokines, was critical for their expansion.

As a consequence of the lack of FceRI $\gamma$ chain, and in line with previous reports $(6,12)$, we found that freshly isolated memory NK cells displayed lower levels of FceRI $\gamma$-coupled activating receptors, such as NKp46 and CD16, and of PLZF transcription factor, whose deficiency is responsible for the silencing of FceRI $\gamma$ 

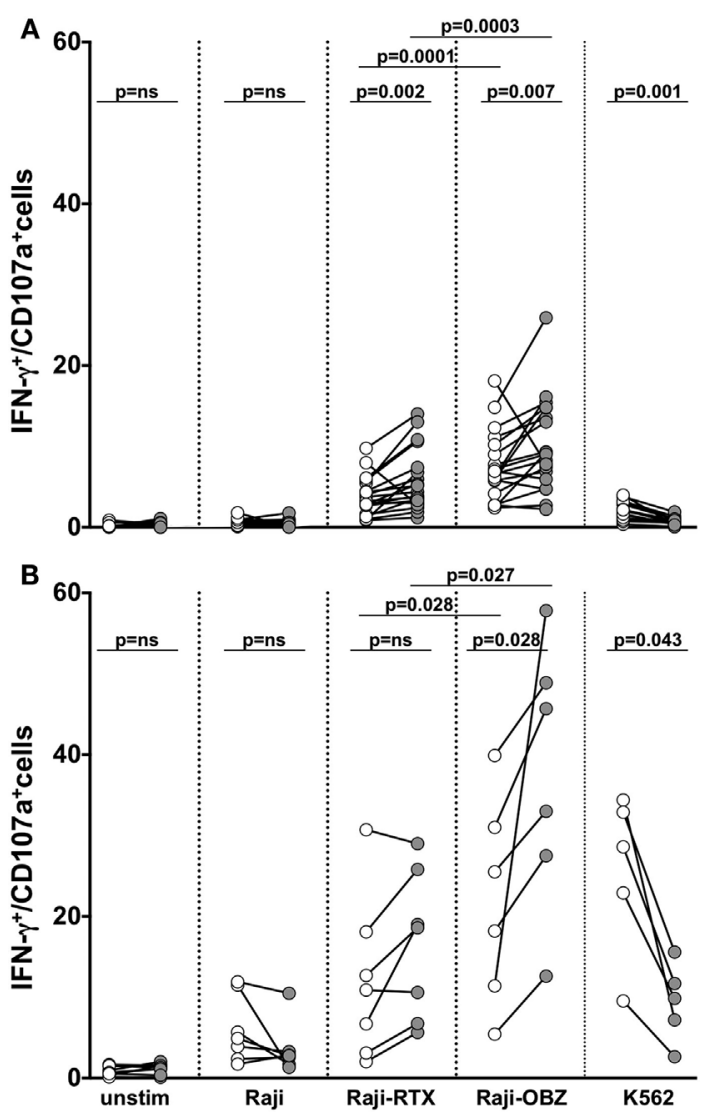

FIGURE 5 | Functional responsiveness of fresh and in vitro cultured memory natural killer (NK) cells. Fresh (A) and cultured (B) memory (filled symbols) and conventional (empty symbols) NK cells were stimulated as indicated, and the percentage of CD107a/ IFN- $\gamma^{+}$was evaluated by immunostaining and FACS analysis. $p$ values of pairwise comparisons are from Wilcoxon non-parametric test.

gene (8), as compared to their conventional counterpart; moreover, as previously reported $(4,10,11), \mathrm{NKG}_{2} \mathrm{C}^{+}$cells were more abundant within memory NK cell pool. Although the expression of these markers was dramatically modulated upon in vitro culture, their distinctive expression pattern on memory $v s$ conventional NK cells was remarkably maintained. Interestingly, our results identify the lower expression of PD-L1, ligand of PD-1 immune checkpoint, as another distinctive feature of freshly isolated memory NK cells, as compared with conventional counterpart. In this regard, although the presence of $\mathrm{PD}^{+} \mathrm{NK}$ cells in a limited percentage of $\mathrm{HCMV}^{+}$healthy individuals has been previously reported (43), the expression of PD-L1 ligand on human NK cells remains mostly unexplored.

Here, we have addressed the impact of CD16 affinity ligation on memory NK cell in vitro and in vivo expansion, and functional activation. We observed that CD16F158V polymorphism dictating receptor binding affinity for $\mathrm{Ab} F \mathrm{~F}$ region $(30,31)$ does not affect the frequency of memory NK cells in vivo, in an extended cohort of CD16 genotyped individuals. Nevertheless, CD16 expression levels on either total NK cells or memory NK cell fraction were higher in low (F/F) with respect to high (V/V) affinity individuals, probably as a consequence of a reduced rate of Ab-induced CD16 internalization. Interestingly, in vitro memory NK cell expansion was heavily affected by CD16 ligation conditions promoted by anti-CD20 therapeutic mAbs. In fact, rituximab, a low-affinity ligand for CD16, much more efficiently drove memory NK cell expansion than obinutuzumab, whose glycoengineering allows a high-affinity interaction with the receptor. The lower capability of obinutuzumab to trigger memory NK cell proliferation was maintained across all CD16 allotypes, in accordance with its capacity to overcome polymorphism-dependent CD16 affinity differences $(37,44)$. Along this line, the rate of memory NK cell expansion in response to rituximab was superior in F/F lowaffinity homozygous with respect to $\mathrm{V} / \mathrm{F}$ donors, suggesting that their proliferation may benefit from a low-affinity CD16 ligation.

Proliferation of both memory and conventional NK cells was negatively affected by the presence of obinutuzumab, when compared with rituximab reference $m A b$. These data suggest that ligation conditions alter the balance between cell death and proliferation stimulated by CD16 cross-linking. Indeed, the capability of CD16-induced signals to induce apoptosis in NK cells has been earlier reported $(19,45)$. Such observation may offer an explanation for the protracted reduction of circulating NK cell levels observed in obinutuzumab-receiving patients (46). Moreover, these data imply that CD16-generated signaling pathways, although not necessary to drive their in vitro expansion, may also operate in conventional NK cells. Co-culture with non-glycoengineered obinutuzumab (GA101-WT)-opsonized targets only partially restored memory NK cell expansion ability, suggesting that other factors than higher affinity for CD16 are responsible for obinutuzumab reduced ability to support memory NK cell proliferation. Indeed, as a class II $\mathrm{mAb}$, it is endowed with a reduced capacity to induce CD20 down-regulation on target cells and a higher capability to induce direct target cells death (47) that could impact stimulation efficiency. However, it is also possible that different CD16 affinity ligation conditions impact on memory NK cell expansion kinetics.

A key feature of memory NK cells is their superior Ab-dependent functional response, with respect to conventional ones $(6-9,22)$. Indeed, despite the lower CD16 expression, they have been shown to more efficiently mediate multifunctional responses, i.e., degranulation and IFN- $\gamma$ production, upon stimulation via ADCC. This characteristic is confirmed by our data, as either freshly isolated or in vitro cultured memory NK cells displayed a higher frequency of multifunctional (IFN $\left.-\gamma^{+} \mathrm{CD} 107 \mathrm{a}^{+}\right)$ cells, upon triggering with anti-CD20-opsonized target cells. The apparent conflict between higher CD16-triggered functional responses and lower surface receptor levels may be explained by the exclusive coupling of CD16 to CD3 $\zeta$ chain in memory NK cells that, thanks to ITAM motif quantitative differences (3 ITAM in CD3 $\zeta$ vs 1 ITAM in FceRI $\gamma$ ), may lead to more robust and efficient biochemical signals (15). Moreover, the residual levels of $\mathrm{CD} 3 \zeta$ chain may preserve the $\mathrm{CD} 2 / \mathrm{CD} 58$ co-stimulatory interaction (48).

Interestingly, stimulation with obinutuzumab-coated targets promoted a more efficient activation of either fresh or in vitro expanded memory NK cells and led to enhanced degranulation and IFN- $\gamma$ production, with respect to rituximab. The enhanced 
obinutuzumab-triggered response, as expected, was also observed in conventional NK cell populations. These data highlight a signal dichotomy downstream of CD16 engagement, being the highaffinity ligation an added value for functional activation and the low affinity useful for proliferative signals. The reduction of NKp46 levels may explain the reduced ability of fresh and in vitro cultured memory NK cell to mediate effector functions in response to stimulation with K562 target cell, being its recognition largely dependent on this receptor (49). However, $\mathrm{NKG}_{2} \mathrm{C}^{+}$memory $\mathrm{NK}$ cells from HCMV-reactivating patients efficiently produced IFN- $\gamma$ upon K562 stimulation $(5,50)$, indicating that the up-regulation of other activating receptors may compensate for the NKp46 defect.

In conclusion, we add new insights on the molecular factors governing the expansion and activation of memory NK cells. In a therapeutic perspective, they represent a particularly attractive tool of cancer immunotherapy, especially in $\mathrm{mAb}$-based regimens where the enhanced responsiveness to mAb-coated targets could significantly impact therapeutic efficacy. These results may guide future attempts to combine the adoptive transfer of ex vivo expanded memory NK cells, or their in vivo manipulation, with therapeutic $\mathrm{mAb}$ administration.

Finally, it is worth investigating the possible contribution of this long-lived NK cell population to the recently described "vaccinal effect" of therapeutic mAbs. Indeed, thanks to their amplified capability to produce cytokines upon CD16 stimulation, memory NK cells could participate to the development of adaptive anti-tumor immune responses, required for the longterm protection of treated patients (27-29).

\section{ETHICS STATEMENT}

Written informed consent was obtained from blood donors, and both the informed consent form and procedure were approved by

\section{REFERENCES}

1. Rölle A, Brodin P. Immune adaptation to environmental influence: the case of NK cells and HCMV. Trends Immunol (2016) 37:233-43. doi:10.1016/j.it. 2016.01.005

2. Cerwenka A, Lanier LL. Natural killer cell memory in infection, inflammation and cancer. Nat Rev Immunol (2016) 16:112-23. doi:10.1038/nri.2015.9

3. Tesi B, Schlums H, Cichocki F, Bryceson YT. Epigenetic regulation of adaptive NK cell diversification. Trends Immunol (2016) 37:451-61. doi:10.1016/j.it. 2016.04.006

4. Lopez-Vergès S, Milush JM, Schwartz BS, Pando MJ, Jarjoura J, York VA, et al. Expansion of a unique CD57 ${ }^{+} \mathrm{NKG} 2 \mathrm{Chi}$ natural killer cell subset during acute human Cytomegalovirus infection. Proc Natl Acad Sci U S A (2011) 108:14725-32. doi:10.1073/pnas.1110900108

5. Foley B, Cooley S, Verneris MR, Pitt M, Curtsinger J, Luo X, et al. Cytomegalovirus reactivation after allogeneic transplantation promotes a lasting increase in educated NKG2C+ natural killer cells with potent function. Blood (2012) 119:2665-74. doi:10.1182/blood-2011-10-386995

6. Hwang I, Zhang T, Scott JM, Kim AR, Lee T, Kakarla T, et al. Identification of human NK cells that are deficient for signaling adaptor FcR $\gamma$ and specialized for antibody-dependent immune functions. Int Immunol (2012) 24:793-802. doi:10.1093/intimm/dxs080

7. Zhang T, Scott JM, Hwang I, Kim S. Cutting edge: antibody-dependent memory-like NK cells distinguished by FcR $\gamma$ deficiency. J Immunol (2013) 190:1402-6. doi:10.4049/jimmunol.1203034

8. Schlums H, Cichocki F, Tesi B, Theorell J, Beziat V, Holmes TD, et al. Cytomegalovirus infection drives adaptive epigenetic diversification of NK cells the Ethics Committee of Sapienza University of Rome. The study was conducted in accordance with the Declaration of Helsinki.

\section{AUTHOR CONTRIBUTIONS}

RG and GP planned the study, analyzed data, and wrote the manuscript. CC and SB performed the experiments, analyzed the data, provided critical inputs, and contributed to writing of the manuscript. CP and LF performed the experiments, analyzed the data, and provided critical inputs. SM and OT provided experimental and critical expertises. AS provided critical inputs and contributed to discussion. All authors contributed to the editing of the article and gave approval.

\section{ACKNOWLEDGMENTS}

We gratefully thank Dr. Claudia Zompetta (Department of Experimental Medicine, Sapienza University of Rome) for technical expertise.

\section{FUNDING}

This study received grants from Italian Ministry for University and Research (MIUR) SIR 2014 (RBSI14022M) (CC), Sapienza University of Rome (Ateneo) (SM), and Italian Association for Cancer Research (AIRC and AIRC $5 \times 1000)($ AS). SB is a recipient of an AIRC fellowship.

\section{SUPPLEMENTARY MATERIAL}

The Supplementary Material for this article can be found online at https://www.frontiersin.org/articles/10.3389/fimmu.2018.01031/ full\#supplementary-material.

with altered signaling and effector function. Immunity (2015) 42:443-56. doi:10.1016/j.immuni.2015.02.008

9. Lee J, Zhang T, Hwang I, Kim A, Nitschke L, Kim M, et al. Epigenetic modification and antibody-dependent expansion of memory-like NK cells in human Cytomegalovirus-infected individuals. Immunity (2015) 42:431-42. doi:10.1016/j.immuni.2015.02.013

10. Muntasell A, Pupuleku A, Cisneros E, Vera A, Moraru M, Vilches C, et al. Relationship of NKG2C copy number with the distribution of distinct Cytomegalovirus-induced adaptive NK cell subsets. JImmunol (2016) 196:3818-27. doi:10.4049/jimmunol.1502438

11. Gumá M, Budt M, Sáez A, Brckalo T, Hengel H, Angulo A, et al. Expansion of CD94/NKG2C+ NK cells in response to human Cytomegalovirus-infected fibroblasts. Blood (2006) 107:3624-31. doi:10.1182/blood-2005-09-3682

12. Rölle A, Pollmann J, Ewen EM, Le VT, Halenius A, Hengel H, et al. IL-12producing monocytes and HLA-E control HCMV-driven NKG2C+ NK cell expansion. J Clin Invest (2014) 124:5305-16. doi:10.1172/JCI77440

13. Béziat V, Dalgard O, Asselah T, Halfon P, Bedossa P, Boudifa A, et al. CMV drives clonal expansion of NKG2C+ NK cells expressing self-specific KIRs in chronic hepatitis patients. Eur J Immunol (2012) 42:447-57. doi:10.1002/ eji.201141826

14. Zhou J, Amran FS, Kramski M, Angelovich TA, Elliott J, Hearps AC, et al. An NK cell population lacking FcR $\gamma$ is expanded in chronically infected HIV patients. J Immunol (2015) 194:4688-97. doi:10.4049/jimmunol.1402448

15. Trinchieri G, Valiante N. Receptors for the Fc fragment of IgG on natural killer cells. Nat Immun (1993) 12:218-34.

16. Long EO, Kim HS, Liu D, Peterson ME, Rajagopalan S. Controlling natural killer cell responses: integration of signals for activation and inhibition. Annu 
Rev Immunol (2013) 31:227-58. doi:10.1146/annurev-immunol-020711075005

17. Martín-Fontecha A, Thomsen LL, Brett S, Gerard C, Lipp M, Lanzavecchia A, et al. Induced recruitment of NK cells to lymph nodes provides IFN-gamma for T(H)1 priming. Nat Immunol (2004) 5:1260-5. doi:10.1038/ni1138

18. Schuster IS, Coudert JD, Andoniou CE, Degli-Esposti MA. "Natural regulators": NK cells as modulators of T cell immunity. Front Immunol (2016) 7:235. doi:10.3389/fimmu.2016.00235

19. Warren HS, Kinnear BF. Quantitative analysis of the effect of CD16 ligation on human NK cell proliferation. J Immunol (1999) 162:735-42.

20. Lee HR, Son CH, Koh EK, Bae JH, Kang CD, Yang K, et al. Expansion of cytotoxic natural killer cells using irradiated autologous peripheral blood mononuclear cells and anti-CD16 antibody. Sci Rep (2017) 7:11075. doi:10.1038/ s41598-017-09259-1

21. Letourneur O, Kennedy IC, Brini AT, Ortaldo JR, O'Shea JJ, Kinet JP. Characterization of the family of dimers associated with $\mathrm{Fc}$ receptors (Fc epsilon RI and Fc gamma RIII). J Immunol (1991) 147:2652-6

22. Liu LL, Landskron J, Ask EH, Enqvist M, Sohlberg E, Traherne JA, et al. Critical role of CD2 co-stimulation in adaptive natural killer cell responses revealed in NKG2C-deficient humans. Cell Rep (2016) 15:1088-99. doi:10.1016/j. celrep.2016.04.005

23. Luetke-Eversloh M, Hammer Q, Durek P, Nordström K, Gasparoni G, Pink M, et al. Human Cytomegalovirus drives epigenetic imprinting of the IFNG locus in NKG2Chi natural killer cells. PLoS Pathog (2014) 10:e1004441. doi:10.1371/ journal.ppat.1004441

24. Taylor RP, Lindorfer MA. Immunotherapeutic mechanisms of anti-CD20 monoclonal antibodies. Curr Opin Immunol (2008) 20:444-9. doi:10.1016/j. coi.2008.05.011

25. Weiner GJ. Building better monoclonal antibody-based therapeutics. Nat Rev Cancer (2015) 15:361-70. doi:10.1038/nrc3930

26. Marshall MJE, Stopforth RJ, Cragg MS. Therapeutic antibodies: what have we learnt from targeting CD20 and where are we going? Front Immunol (2017) 8:1245. doi:10.3389/fimmu.2017.01245

27. Battella S, Cox MC, Santoni A, Palmieri G. Natural killer (NK) cells and anti-tumor therapeutic mAb: unexplored interactions. J Leukoc Biol (2016) 99:87-96. doi:10.1189/jlb.5VMR0415-141R

28. Abès R, Gélizé E, Fridman WH, Teillaud JL. Long-lasting antitumor protection by anti-CD20 antibody through cellular immune response. Blood (2010) 116:926-34. doi:10.1182/blood-2009-10-248609

29. Pampena MB, Levy EM. Natural killer cells as helper cells in dendritic cell cancer vaccines. Front Immunol (2015) 6:13. doi:10.3389/fimmu.2015.00013

30. Cartron G, Dacheux L, Salles G, Solal-Celigny P, Bardos P, Colombat P, et al. Therapeutic activity of humanized anti-CD20 monoclonal antibody and polymorphism in IgG Fc receptor FcgammaRIIIa gene. Blood (2002) 99:754-8. doi:10.1182/blood.V99.3.754

31. Persky DO, Dornan D, Goldman BH, Braziel RM, Fisher RI, Leblanc M, et al. Fc gamma receptor 3a genotype predicts overall survival in follicular lymphoma patients treated on SWOG trials with combined monoclonal antibody plus chemotherapy but not chemotherapy alone. Haematologica (2012) 97:937-42. doi:10.3324/haematol.2011.050419

32. Goede V, Fischer K, Busch R, Engelke A, Eichhorst B, Wendtner CM, et al. Obinutuzumab plus chlorambucil in patients with CLL and coexisting conditions. N Engl J Med (2014) 370:1101-10. doi:10.1056/NEJMoa1313984

33. Sehn LH, Chua N, Mayer J, Dueck G, Trněný M, Bouabdallah K, et al. Obinutuzumab plus bendamustine versus bendamustine monotherapy in patients with rituximab-refractory indolent non-Hodgkin lymphoma (GADOLIN): a randomised, controlled, open-label, multicentre, phase 3 trial. Lancet Oncol (2016) 17:1081-93. doi:10.1016/S1470-2045(16)30097-3

34. Marcus R, Davies A, Ando K, Klapper W, Opat S, Owen C, et al. Obinutuzumab for the first-line treatment of follicular lymphoma. N Engl J Med (2017) 377:1331-44. doi:10.1056/NEJMoa1614598

35. Klein C, Lammens A, Schäfer W, Georges G, Schwaiger M, Mössner E, et al. Epitope interactions of monoclonal antibodies targeting CD20 and their relationship to functional properties. MAbs (2013) 5:22-33. doi:10.4161/mabs.22771

36. Capuano C, Romanelli M, Pighi C, Cimino G, Rago A, Molfetta R, et al. Anti-CD20 therapy acts via Fc $\gamma$ RIIIA to diminish responsiveness of human natural killer cells. Cancer Res (2015) 75:4097-108. doi:10.1158/0008-5472. CAN-15-0781

37. Capuano C, Pighi C, Molfetta R, Paolini R, Battella S, Palmieri G, et al. Obinutuzumab-mediated high-affinity ligation of Fc $\gamma$ RIIIA/CD16 primes NK cells for IFN $\gamma$ production. Oncoimmunology (2017) 6:e1290037. doi:10.1080/2162402X.2017.1290037

38. Böttcher S, Ritgen M, Brüggemann M, Raff T, Lüschen S, Humpe A, et al. Flow cytometric assay for determination of FcgammaRIIIA-158 V/F polymorphism. J Immunol Methods (2005) 306:128-36. doi:10.1016/j.jim.2005.08.004

39. Guillerey C, Huntington ND, Smyth MJ. Targeting natural killer cells in cancer immunotherapy. Nat Immunol (2016) 17:1025-36. doi:10.1038/ni.3518

40. Granzin M, Wagner J, Köhl U, Cerwenka A, Huppert V, Ullrich E. Shaping of natural killer cell antitumor activity by Ex vivo cultivation. Front Immunol (2017) 8:458. doi:10.3389/fimmu.2017.00458

41. Kim TJ, Kim M, Kim HM, Lim SA, Kim EO, Kim K, et al. Homotypic NK cellto-cell communication controls cytokine responsiveness of innate immune NK cells. Sci Rep (2014) 4:7157. doi:10.1038/srep07157

42. Liu LL, Béziat V, Oei VYS, Pfefferle A, Schaffer M, Lehmann S, et al. Ex vivo expanded adaptive NK cells effectively kill primary acute lymphoblastic leukemia cells. Cancer Immunol Res (2017) 5:654-65. doi:10.1158/2326-6066. CIR-16-0296

43. Pesce S, Greppi M, Tabellini G, Rampinelli F, Parolini S, Olive D, et al. Identification of a subset of human natural killer cells expressing high levels of programmed death 1: a phenotypic and functional characterization. J Allergy Clin Immunol (2017) 139:335-46. doi:10.1016/j.jaci.2016.04.025

44. Terszowski G, Klein C, Stern M. KIR/HLA interactions negatively affect rituximab- but not GA101 (obinutuzumab)-induced antibody- dependent cellular cytotoxicity. J Immunol (2014) 192:5618-24. doi:10.4049/jimmunol. 1400288

45. Ortaldo JR, Mason AT, O’Shea JJ. Receptor-induced death in human natural killer cells: involvement of CD16. J Exp Med (1995) 181:339-44. doi:10.1084/ jem.181.1.339

46. Freeman CL, Morschhauser F, Sehn L, Dixon M, Houghton R, Lamy T, et al. Cytokine release in patients with CLL treated with obinutuzumab and possible relationship with infusion-related reactions. Blood (2015) 126:2646-9. doi:10.1182/blood-2015-09-670802

47. Tipton TR, Roghanian A, Oldham RJ, Carter MJ, Cox KL, Mockridge CI, et al. Antigenic modulation limits the effector cell mechanisms employed by type I anti-CD20 monoclonal antibodies. Blood (2015) 125:1901-9. doi:10.1182/ blood-2014-07-588376

48. Grier JT, Forbes LR, Monaco-Shawver L, Oshinsky J, Atkinson TP, Moody C, et al. Human immunodeficiency-causing mutation defines CD16 in spontaneous NK cell cytotoxicity. J Clin Invest (2012) 122:3769-80. doi:10.1172/ JCI64837

49. Sivori S, Pende D, Bottino C, Marcenaro E, Pessino A, Biassoni R, et al. NKp46 is the major triggering receptor involved in the natural cytotoxicity of fresh or cultured human NK cells. Correlation between surface density of NKp46 and natural cytotoxicity against autologous, allogeneic or xenogeneic target cells. Eur J Immunol (1999) 29:1656-66. doi:10.1002/ (SICI)1521-4141(199905)29:05<1656::AID-IMMU1656>3.0.CO;2-1

50. Cichocki F, Cooley S, Davis Z, DeFor TE, Schlums H, Zhang B, et al. CD56dimCD57+NKG2C+ NK cell expansion is associated with reduced leukemia relapse after reduced intensity HCT. Leukemia (2016) 30:456-63. doi:10.1038/leu.2015.260

Conflict of Interest Statement: The submitted work was carried out in the absence of any personal, professional, or financial relationships that could potentially be construed as a conflict of interest.

Copyright (C) 2018 Capuano, Battella, Pighi, Franchitti, Turriziani, Morrone, Santoni, Galandrini and Palmieri. This is an open-access article distributed under the terms of the Creative Commons Attribution License (CC BY). The use, distribution or reproduction in other forums is permitted, provided the original author(s) and the copyright owner are credited and that the original publication in this journal is cited, in accordance with accepted academic practice. No use, distribution or reproduction is permitted which does not comply with these terms. 